\title{
Transfer Learning via Artificial Intelligence for Guiding Implant Placement in the Posterior Mandible: an in vitro Study
}

\section{Yun Liu}

Sun Yat-sen University

\section{Zhi-cong Chen}

Sun Yat-sen University

\section{Chun-ho Chu}

Sun Yat-sen University

Fei-Long Deng ( $\nabla$ dengfl@mail.sysu.edu.cn )

Sun Yat-sen University

\section{Research Article}

Keywords: artificial intelligence, SSD, V2V-PoseNet, algorithm, implant position

Posted Date: October 26th, 2021

DOI: https://doi.org/10.21203/rs.3.rs-986672/v1

License: (c) (i) This work is licensed under a Creative Commons Attribution 4.0 International License. Read Full License 


\section{Abstract}

Background: To explore the capacity of a single shot multibox detector (SSD) and Voxel-to-voxel prediction network for pose estimation (V2V-PoseNet) based artificial intelligence (Al) system in automatically designing implant plan.

Methods: 2500 and 67 cases were used to develop and pre-train the Al system. After that, 12 patients who missed the mandibular left first molars were selected to test the capacity of the Al in automatically designing implant plan. There were three algorithms-based implant positions. They are Group A, B and C (8, 9 and 10 points dependent implant position, respectively). The Al system was then used to detect the characteristic annotators and determine the implant position. For every group, the actual implant position was compared with the algorithm-determined ideal position. And global, angular, depth and lateral deviation were calculate. One-way ANOVA followed by Tukey's test was performed for statistical comparisons. The significance value was set at $P<0.05$.

Results: Group C represented the least coronal $(0.6638 \pm 0.2651$, range: 0.2060 to $1.109 \mathrm{~mm})$ and apical $(1.157 \pm 0.3350$, range: 0.5840 to $1.654 \mathrm{~mm})$ deviation, the same trend was observed in the angular deviation (5.307 $\pm 2.891^{\circ}$, range: 2.049 to $10.90^{\circ}$ ), and the results are similar with the traditional statistic guide.

Conclusion: It can be concluded that the Al system has the capacity of deep learning. And as more characteristic annotators be involved in the algorithm, the Al system can figure out the anatomy of the object region better, then generate the ideal implant plan via deep learning algorithm.

\section{Background}

Optimal three-dimensional (3D) position of dental implants is the key for functional and aesthetic outcomes ${ }^{1,2}$. Virtual implantation improves the accuracy of implant placement. Subsequently, guided surgery opens up possibilities to transfer the $3 \mathrm{D}$ pre-surgical planning to implant surgery, to achieve ideal $3 \mathrm{D}$ implant placement ${ }^{1-4}$.

As surgical templates can reduce human error, guided surgery has come to play an important role in precise bone drilling and implant placement ${ }^{4-6}$. However, traditional surgical templates still have several disadvantages, including extensive manual work for preoperative planning and increased cost of template fabrication for each case. Besides that, the accuracy of guided implant surgery depends on the dentist and technician, while there are learning curves and expenses related to training ${ }^{7}$. Our approach to improve the traditional surgical template is the use of artificial intelligence (Al).

Al refers to a serious of technologies that allow computers and machines to imitate human intelligence ${ }^{8}$. It has come to play an important role in healthcare, including analyzing a diverse array of patient data and simulating human logic to perform some tasks ${ }^{9-12}$. Al iteratively learn the intrinsic statistics underlying the pairing data and algorithm, to make plan on unseen data ${ }^{8,10}$. Recently, there has been a significantly increasing tendency in the number of $\mathrm{Al}$ studies in medicine, mostly for disease detection and classification purposes $8,10,13-15$. And the use of Al for radiological images reading has been widely explored ${ }^{12,13,16}$.

Al system would enable all clinicians to design and practice the complicate cases at the same level of practical expertise as the very best clinicians. Since database could be shared between physicians without the privacy risks of leaking patient data, there is almost limitless potential to renew the system learned from the diverse physicians and diverse patients ${ }^{11,17}$. 
Recently, Al technique has also been used in dentistry for various purposes $8,18,19$. For instance, Shintaro et al. ${ }^{20}$ used $^{2}$ Al to accurately classify dental implant brands from various panoramic X-ray images, Joel et al. ${ }^{21}$ presented a deep learning system for mandibular canal segmentation, Jin-Ju et al. ${ }^{22}$ used Al for automatic 3D analysis of bone alteration after maxillary sinus augmentation. Jun et al. ${ }^{16}$ developed a deep neural network to determine the periimplant marginal bone loss. The accuracies for most of the tasks are promising.

The usage of Al for dental diagnostics has been developed quickly in the recent years ${ }^{8,18,23}$. It worth further focusing on the possibilities of automatically generated implant surgery plans. To our knowledge, there were rare studies in this field. This study explored a deep learning model, named SSD ${ }^{24}$, with V2V-PoseNet ${ }^{25}$ as its backbone architecture, to automatically recognize the target region, detect the bone contour and capture the characteristic annotators, then generate the implant plan. Then we assessed the accuracy of the automatically designed templates, which satisfies the well-established algorithm.

The purpose of this in vitro study was to explore the capacity of the Al system in automatically designing implant plan, through deep learning. And compare the accuracy of 8, 9 and 10 characteristic annotators based algorithm guided surgery in posterior mandible.

\section{Methods}

All patients consented to the use of their data for research purposes and signed informed consent forms accordingly. This study protocol was approved by the ethical committees of the Guanghua School of Stomatology, Hospital of Stomatology, Sun Yat-sen University (KQEC-2020-063-01).

\subsection{Sample}

The datasets were divided into pre-training, training and test sets. The pre-training set, including 2500 various edentulous sites undergoing CBCT image (2018.10-2020.10), were used to train and develop the model parameters. After that, the model hyper parameters and architecture selected using the training set, which includes 67 cases who undergo CBCT and intraoral scanning. The selected 67 patients should meet the following conditions: 1) just miss the mandibular left first molar; 2) with the normal occlusion; 3) without any metallic prosthesis; 4$) \geq 18$ years old. Finally, 12 patients undergoing CBCT (NewTom VGi CBCT imaging unit, Verona, Italy) and intraoral scanning (SWEDEN \& MARTINA, Veneto, Italy) who met the above criteria were collected for the following automatically designed implant surgery guide generation. To avoid over-optimistic results because of over-fitting, that is, memorizing specific features of edentulous sites, each of the three sets had an independent set of patients.

\subsection{Software workflow}

The Al software workflow is presented in Fig. 1. A model named SSD ${ }^{24}$ has been proposed for edentulous site and related key points detection.

\subsubsection{Evaluation of edentulous area condition}

The image flow chart of automatically implant position design is shown in Figure 2. SSD model, a convolutional neural network (CNN), was used to construct the Al system. All files were uploaded to SSD for evaluation of edentulous area condition. First, the software automatically generated a panoramic image and detect the missing tooth position through CBCT (DICOM data) reading. Second, the region of interest (ROI) was constructed. All slices were extracted from ROI of the panoramic image ribbon and a small box area was cut out from the DICOM data for 
processing. Third, detect the bone contour and capture the designed characteristic annotators based on V2V-PoseNet. Then the implant axis was generated. Finally, the implant position was created automatically.

\subsection{2 haracteristic annotators capture}

The characteristic annotators were recorded based on the condition of the alveolar bone 3D features, the neighboring teeth and the opposite teeth (Fig. 3). They are: Point 1 (P1): The buccal junction point between the alveolar ridge of the missing tooth and the adjacent anterior tooth; Point 2 (P2): The buccal middle point between the alveolar ridge of the adjacent anterior and posterior teeth; Point 3 (P3): The buccal junction point between the alveolar ridge of the missing tooth and the adjacent posterior tooth; Point 4 (P4): The lingual junction point between the alveolar ridge of the missing tooth and the adjacent anterior tooth; Point 5 (P5): The lingual middle point between the alveolar ridge of the adjacent anterior and posterior teeth; Point 6 (P6): The lingual junction point between the alveolar ridge of the missing tooth and the adjacent posterior tooth; Point 7 (P7): The height of the contour of the adjacent anterior tooth approaching to the target site; Point 8 (P8): The height of the contour of the adjacent posterior tooth approaching to the target site; Point 9 (P9): The function cusp of its opposite teeth; Point 10 (P10): The central fossa of its opposite teeth.

\subsubsection{Implantation plan generation}

All of the characteristic points were detected using V2V-PoseNet method. The characteristic points provided information about the implant position. In brief, there are three measurements. The first measurement showed the central spot of the implant neck $\left(P_{a}\right)$, the second measurement showed the axial feature spot $\left(P_{b}\right)$, and the third measurement showed the implant axis (Fig. 4). The linkage between $P_{a}$ and $P_{b}$ represented the implant axis. Besides that, the inferior alveolar nerve were marked by the operator. All the planed implants were bone level implants (NobelReplace CC; Nobelbiocare) of $4.3 \mathrm{~mm} \times 10 \mathrm{~mm}$ or $4.3 \mathrm{~mm} \times 11.5 \mathrm{~mm}$. The implant length was determined by the operator, to keep a safe distance $(\geq 2 \mathrm{~mm})$ from the inferior alveolar nerve.

There are three kinds of calculation methods for the three measurements ( $P_{a}, P_{b}$ and implant axis). Accordingly, for every case, there are three groups of implantation plans. They are (1) Group A: 8 points (P1-P8) dependent implant position; (2) Group B: 9 points (P1-P9) dependent implant position; (3) Group C: 10 points (P1-P10) dependent implant position. In each group, $P_{a}$ and $P_{b}$ follow specific algorithm (Table 1 ).

Table 1. The specific algorithm for $P_{a}$ and $P_{b}$

\begin{tabular}{ccc}
\hline Group & $P_{a}$ & $P_{b}$ \\
\hline $\mathrm{A}$ & $\frac{\sum_{i=0}^{6} P_{i}}{6}$ & $\frac{\sum_{i=7}^{8} P_{i}}{2}$ \\
\hline $\mathrm{B}$ & $\sum_{i=0}^{6} P_{i}$ & $\frac{\sum_{i=7}^{9} P_{i}}{6}$ \\
\hline $\mathrm{C}$ & $\frac{\sum_{i=0}^{6} P_{i}}{6}$ & $\frac{\sum_{i=7}^{10} P_{i}}{4}$ \\
\hline
\end{tabular}




\subsubsection{Surgical guides fabrication}

Based on the plan, implant surgical templates were fabricated from ultraviolet sensitive liquid resin E-Dent (EnvisionTEC). Each surgical guide was $3 \mathrm{~mm}$ thick. All the templates were teeth-supported and full guided. And all the templates were equipped with metal sleeves to serve as the guidance for drill key. According to the 12 partially edentulous cases, physical surgical resin models (B9R-1-RED; B9Creations) were 3D printed. For each guide and surgical model, one implant was inserted on the basis of the fully guided surgical protocol.

The implant site preparation and implant placement were performed using commercial surgical guide kits (Nobel Surgical Kit). Once the templates were properly fitted on the in vitro models, all the drilling steps and implant placement took place without removing the guide. The manufacturer's recommendations were followed through the process of implant site preparation and implant placement, to avoid unpredictable deviation. After the osteotomy, a planned NobelReplace CC implant was placed with the guided portable adaptor. And all the surgeries were accomplished by the same clinician, to estimate the intra-observer variability.

\subsubsection{Accuracy evaluation}

Following implant placement, specific implant scan body was used for accuracy evaluation. In brief, the scan body was attached on the implant, and intraoral scanning was performed to obtain STL data. Then the reverse engineering software (Geomagic Studio; Raindrop Geomagic) was used to analyze the deviation between the implant position and the algorithm bases ideal implant position.

For every group, the virtual implant position according to the various algorithm was marked for reference. Through aligning the data sets, the actual implant position was compared with the algorithm-determined position, and the deviations were performed in $3 \mathrm{D}^{26}$ (Fig. 5). Besides, perforations of the apical buccal/lingual bone were assessed.

The angular deviation was defined as the angle between the centerline of the actual and virtual algorithm-determined implant (a). The global deviation was calculated as the 3D distance of apical/coronal center between the placed and algorithm-determined implant. The global deviation was subdivided into the lateral deviation (perpendicular to it) and the depth deviation (along the implant axis). Moreover, the lateral deviation was split into the bucco-lingual deviation (along the bucco-lingual axis) and the mesio-distal deviation (perpendicular to the bucco-lingual axis). Furthermore, to illustrate the deviations in exact directions, a positive value was used when the placed implant was mesial/buccal/apical to the virtual algorithm-determined implant, while a negative value corresponded to distal/lingual/coronal placement compared with the algorithm-determined position. For this study, all the measurements were accomplished by one observer.

\subsubsection{Statistics}

All quantitative data are presented as means \pm SD. One-way analysis of variance (ANOVA) followed by Tukey's test was performed for statistical comparisons. The significance value was set at $P<0.05$. All data were analyzed with SPSS statistics v25.0. (IBM Corporation, Armonk, NY, USA).

\section{Results}

The parameters for the three different groups are shown in Tables 2 and 3 . The tables illustrating the differences among the groups are presented in Figure 6A-F. In Table 2, the global (apical and coronal), depth and angular deviations are shown. According to the statistical tests, Group $\mathrm{C}$ represented the best in accuracy. Group $\mathrm{C}$ 
represented significantly lower apical global deviation $(1.157 \pm 0.3350)$ than Group A $(1.717 \pm 0.3355)(P=0.0045)$. The same trends were observed in coronal global deviation and depth deviation, with no significant difference. And for angle deviation, Group B (4.424 \pm 1.505$)$ represented the best, however, no significant difference was shown.

The lateral, bucco-lingual (apical and coronal), and mesio-distal (apical and coronal) deviations were shown in Table 3. Group $C$ represented the best in accuracy. The apical bucco-lingual deviations were significantly smaller in Group $C$ in both buccal direction $(0.6377 \pm 0.5287, P=0.0103)$ and absolute value $(0.7149 \pm 0.4069, P=0.0011)$ when compared with Group A. Group C represent the same trend in the lateral, coronal bucco-lingual, coronal mesio-distal, and apical mesio-distal (mesial direction) deviations, with no significant difference. And for the mesio-distal (absolute value) deviation, Group B $(4.424 \pm 1.505)$ represented the best, with no significant difference.

In regard to the exact direction, for all the groups the placed implants were buccal to the algorithm calculated position. Group $C$ represented the least buccal shift both at the apical $(0.6377 \pm 0.5287 \mathrm{~mm})$ and coronal level $(0.2088$ $\pm 0.2523 \mathrm{~mm}$ ). And the difference was significant between Group C and Group A at the apical level $(P=0.0103)$. Moreover, in this study no buccal or lingual bone perforation was found. For mesio-distal deviation, Group $\mathrm{C}$ showed the least shift. And for Group C, no obvious tendency was found either towards mesial or distal, at coronal or apical level. Moreover, there was neither buccal nor lingual perforation in all the groups.

Table 2

Global (coronal and apical), angular, and depth deviations of various group

\begin{tabular}{|c|c|c|c|c|c|c|}
\hline \multicolumn{2}{|c|}{ Deviation type } & \multicolumn{2}{|c|}{ Global deviation } & \multirow[t]{2}{*}{ Angular deviation } & \multicolumn{2}{|l|}{ Depth deviation } \\
\hline & & Coronal & Apical & & Considering direction & Absolute value \\
\hline \multirow[t]{4}{*}{ Group A } & Mean & 0.9077 & 1.717 & 5.073 & -0.2085 & 0.4548 \\
\hline & Min & 0.2590 & 0.8220 & 2.076 & -1.376 & 0.1130 \\
\hline & Max & 1.478 & 2.240 & 9.460 & 0.5740 & 1.376 \\
\hline & SD & 0.3220 & 0.3555 & 2.270 & 0.5605 & 0.3686 \\
\hline \multirow[t]{4}{*}{ Group B } & Mean & 0.8238 & 1.551 & 4.424 & -0.2742 & 0.4295 \\
\hline & Min & 0.2800 & 0.6570 & 1.681 & -1.247 & 0.01440 \\
\hline & Max & 1.332 & 2.354 & 6.860 & 0.5870 & 1.247 \\
\hline & SD & 0.3140 & 0.4905 & 1.505 & 0.5060 & 0.3700 \\
\hline \multirow[t]{4}{*}{ Group C } & Mean & 0.6638 & 1.157 & 5.307 & -0.1886 & 0.3599 \\
\hline & Min & 0.2060 & 0.5840 & 2.049 & -1.106 & 0.04900 \\
\hline & Max & 1.109 & 1.654 & 10.90 & 0.2930 & 1.106 \\
\hline & SD & 0.2651 & 0.3350 & 2.891 & 0.4639 & 0.3358 \\
\hline
\end{tabular}


Table 3

Lateral, bucco-lingual (coronal and apical), and mesio-distal (coronal and apical) deviations of various group

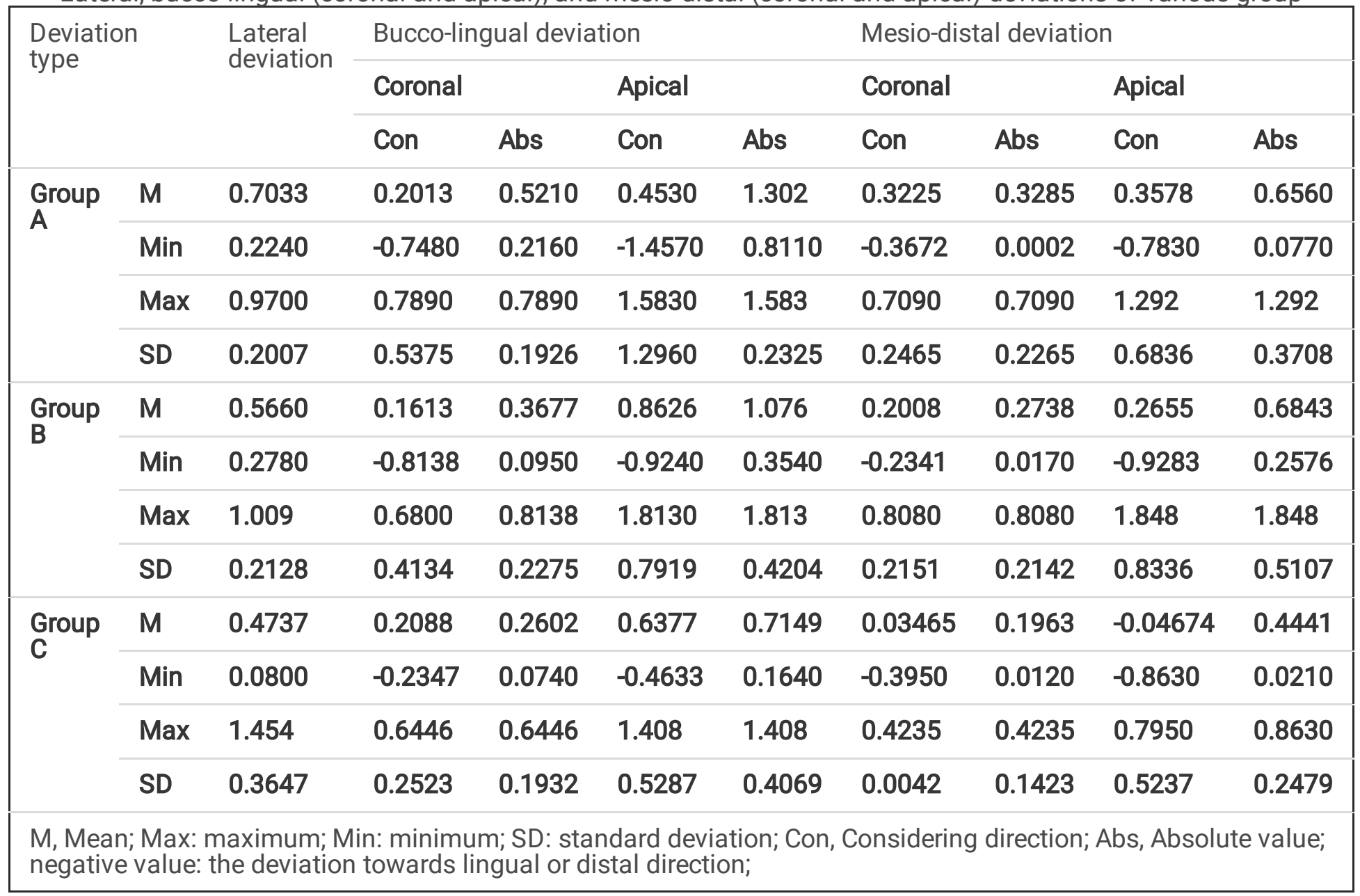

\section{Discussion}

The technology underlying Al applications was machine learning, which iteratively learned the inherent statistical patterns from specific datasets and algorithms ${ }^{5}$. However, as the most effective number of characteristic annotators was unclear, the data used to train the machine was small, and the result generation process was invisible, there were doubts about the accuracy and replicability $8,27$.

This study developed a SSD and V2V-PoseNet based system for image recognition and characteristic points capture, then implant position design. Based on the deep learning frame, the datasets were trained using the designed SSD algorithm to generate image identification and location models. Besides that, V2V-PoseNet method was used to detect the feature of the ROI. After training the image set, 12 independent test images were used to evaluate the trained model. Our Al system showed good performance for the implant position design and the accuracy is similar with the recently reported statistic guide ${ }^{28}$.

Moreover, we found that the number of characteristic annotators influenced the performance of our Al system. A trend of more characteristic annotators induced more accuracy was observed. In brief, Group C performed better than Group A and B, for global (apical and coronal), angular, depth, lateral, bucco-lingual and mesia-distal deviations. In another word, Group C was the most accurate, and it was used to further improve the Al system. This phenomenon reminded us that our Al system was full of learning ability, and more information contributed to the accuracy. 
In Group C, the average deviations were $0.6638 \pm 0.2651$ (range: 0.2060 to $1.109 \mathrm{~mm}$ ) for the coronal deviation, $1.157 \pm 0.3350$ (range: 0.5840 to $1.654 \mathrm{~mm}$ ) for the apical deviation, and $5.307 \pm 2.891^{\circ}$ (range: 2.049 to $10.90^{\circ}$ ) for the angular deviation (Table 2, Fig. 6A-B), and the results are similar with the traditional statistic guide ${ }^{28}$. For instance, Zhaozhao et al. ${ }^{26}$ recently reported $0.85 \mathrm{~mm}$ (range:0.42 to $1.51 \mathrm{~mm}$ ) global deviation at the coronal level, $0.93 \mathrm{~mm}$ (range: 0.64 to $1.72 \mathrm{~mm}$ ) global deviation at the apical level, and $3.11^{\circ}$ (range:0.66 to $4.95^{\circ}$ )for the angular deviation. Vinci et al. ${ }^{6}$ reported $0.43 \mathrm{~mm}$ (range: $0.30-1.77 \mathrm{~mm}$ ) global deviation at the apical level and $0.28 \mathrm{~mm}$ (range: $0.08-$ $1.18 \mathrm{~mm}$ ) at the coronal level.

For the depth deviation, the average deviation was $-0.1886 \pm 0.4639$ for Group C, smaller than Group $A$ $(-0.2085 \pm 0.5605)$ and Group B $(-0.2742 \pm 0.5060)$ (Table 2, Fig. $6 \mathrm{C})$. And for all the three groups, the implants were in a more coronal direction compared to the virtual position. This phenomenon also has been reported by Vercruyssen et $\mathrm{al}^{29}$ and Zhaozhao et $\mathrm{al}^{26}$. However, as more characteristic annotators been calculated, the deviation decreased.

Regarding the bucco-lingual deviation, the implants tended to move buccally both at coronal and apical level, and a greater deviation was found at the apical level. The coronal/apical deviation may occur during the processes of either drilling or implant placement ${ }^{26}$. However, the bucco-lingual deviation was small in Group C (Table 3, Fig. 6E). As to the mesia-distal deviation, for Group C, there were nearly no tendency neither toward mesial nor distal, both at the coronal and apical level (Table 3, Fig. 6F). For Group C, tiny bucco-lingual and mesia-distal deviations would bring mechanical equilibrium, which be beneficial to the long-term success of dental implant.

The high accuracy of our Al system mostly depended on SSD, a deep learning technology based superior convolutional neural network (CNN) algorithm ${ }^{30}$. SSD is one of the best Al models in image identification and location, which consist of at least 16 layers $^{31,32}$. It is an effective object detector for multiple targets recognition within just one stage ${ }^{10}$. Yao-Kuang et al. ${ }^{31}$ used a SSD system for diagnosing esophageal cancer, which showed good diagnostic performance and the accuracy can achieve $90 \%$. Orhan et al. ${ }^{33}$ also reported that the volume calculation with CNN algorithm were compatible with the clinicians.

The high accuracy of our Al system also attributes to the V2V-PoseNet algorithm. It's a 3D CNN put forward by Gyeongsik Moon et $\mathrm{al}^{34}$, which provided accurate estimates. V2V-PoseNet took voxelized grids as inputs and estimated the per-voxel likelihood for the key parameters. This 3D CNN can detect the actual contour of the objects without perspective distortion and estimate the per-voxel likelihood of each parameter, so it can learn the designed task easily ${ }^{34}$.

To sum up, SSD and V2V-PoseNet were used to train the datasets ${ }^{32,35}$, and the deep learning neural network, especially the 10 characteristic annotators based algorithm group, was fairly accurate and clinically reliable. The core of SSD model is to explore objects and corresponding locations in given bounding boxes, realizing object location prediction ${ }^{36}$. It relatively simplified the processing, while increasing the identification.

However, due to the limited test size, the results in this study should be interpreted with caution, and more training images were needed to improve the performance of our system. Besides that, there are some real clinical elements, such as limited view and inter-occlusal distance ${ }^{37}$ that the present in vitro study cannot reflect. Moreover, based on the pronounced learning abilities of the Al system, in the future more key parameters would be incorporated into the algorithm to figure out the best implant plan.

\section{Conclusion}


It can be concluded that the SSD and V2V-PoseNet based Al system has the capacity of deep learning from the data and specific algorithms. And as more characteristic annotators be involved in the algorithm, the Al system can figure out the anatomy of the object region better, then generate the ideal implant plan via specific algorithm.

\section{Abbreviations}

SSD: single shot multibox detector; V2V-PoseNet: Voxel-to-voxel prediction network for pose estimation; Al: artificial intelligence; 3D: three-dimensional; CNN: convolutional neural network; ROI: region of interest.

\section{Declarations}

\section{Ethics approval and consent to participate}

This study was approved by the ethical committees of the Guanghua School of Stomatology, Hospital of Stomatology, Sun Yat-sen University (KQEC-2020-063-01).

\section{Consent for publication}

All patients consented to the use of their data for research purposes and signed informed consent forms accordingly.

\section{Availability of data and materials}

The datasets used and/or analysed during the current study are available from the corresponding author on reasonable request.

\section{Competing interests}

The authors declare that they have no conflict of interests.

\section{Funding}

This work was supported by the Science and Technology Plan of Guangdong Province, People's Republic of China (2017B090912004) and the Applied Basic Research Programs of Science and Technology Department of Guangdong Province, People's Republic of China (2019A1515110514).

\section{Authors' contributions}

Yun Liu: Conceptualization, methodology \& data curation, and figures 5-6 preparing

Zhi-cong Chen: Writing the main manuscript

Chun-ho Chu: Formal analysis and figures 1-4 preparing

Fei-Long Deng: Funding acquisition, tables 1-3 preparing and manuscript review \& editing

All authors read and approved the final manuscript.

\section{Acknowledgements}

We thank all the research staff members at the Department of Oral Implantology, Guanghua School of Stomatology, Sun Yat-sen University. 


\section{References}

1. Abduo J, Lau D. Accuracy of static computer-assisted implant placement in long span edentulous area by novice implant clinicians: A cross-sectional in vitro study comparing fully-guided, pilot-guided, and freehand implant placement protocols. Clin Implant Dent Relat Res. Apr 42021.

2. Wei SM, Zhu Y, Wei JX, Zhang CN, Shi JY, Lai HC. Accuracy of dynamic navigation in implant surgery: A systematic review and meta-analysis. Clin Oral Implants Res. Feb 42021.

3. Wu D, Zhou L, Yang J, Zhang B, Lin Y, Chen J, Huang W, Chen Y. Accuracy of dynamic navigation compared to static surgical guide for dental implant placement. Int J Implant Dent. Nov 24 2020;6(1):78.

4. Varga E, Jr., Antal M, Major L, Kiscsatari R, Braunitzer G, Piffko J. Guidance means accuracy: A randomized clinical trial on freehand versus guided dental implantation. Clin Oral Implants Res. Jan 202020.

5. Gargallo-Albiol J, Salomo-Coll O, Lozano-Carrascal N, Wang HL, Hernandez-Alfaro F. Intra-osseous heat generation during implant bed preparation with static navigation: Multi-factor in vitro study. Clin Oral Implants Res. Feb 242021.

6. Vinci R, Manacorda M, Abundo R, Lucchina AG, Scarano A, Crocetta C, Muzio LL, Gherlone EF, Mastrangelo F. Accuracy of Edentulous Computer-Aided Implant Surgery as Compared to Virtual Planning: A Retrospective Multicenter Study. J Clin Med. Mar 12 2020;9(3).

7. Tatakis DN, Chien HH, Parashis AO. Guided implant surgery risks and their prevention. Periodontol 2000. Oct 2019;81(1):194-208.

8. Schwendicke F, Singh T, Lee JH, Gaudin R, Chaurasia A, Wiegand T, Uribe S, Krois J, network leoh, the ITUWHOfgAlfH. Artificial intelligence in dental research: Checklist for Authors, Reviewers, Readers. J Dent. Feb 22 2021:103610.

9. Wang F, Preininger A. Al in Health: State of the Art, Challenges, and Future Directions. Yearbook of Medical Informatics. 2019;28(01):016-026.

10. Hsiao YP, Chiu CW, Lu CW, Nguyen HT, Tseng YS, Hsieh SC, Wang HC. Identification of Skin Lesions by Using Single-Step Multiframe Detector. J Clin Med. Jan 4 2021;10(1).

11. Norgeot B, Glicksberg BS, Butte AJ. A call for deep-learning healthcare. Nat Med. Jan 2019;25(1):14-15.

12. Topol EJ. High-performance medicine: the convergence of human and artificial intelligence. Nat Med. Jan 2019;25(1):44-56.

13. Bayrakdar SK, Orhan K, Bayrakdar IS, Bilgir E, Ezhov M, Gusarev M, Shumilov E. A deep learning approach for dental implant planning in cone-beam computed tomography images. BMC Med Imaging. May 19 2021;21(1):86.

14. Su C, Xu Z, Pathak J, Wang F. Deep learning in mental health outcome research: a scoping review. Translational Psychiatry. 2020;10(1).

15. Renard F, Guedria S, Palma N, Vuillerme N. Variability and reproducibility in deep learning for medical image segmentation. Sci Rep. Aug 13 2020;10(1):13724.

16. Cha JY, Yoon HI, Yeo IS, Huh KH, Han JS. Peri-Implant Bone Loss Measurement Using a Region-Based Convolutional Neural Network on Dental Periapical Radiographs. J Clin Med. Mar 2 2021;10(5).

17. Miller DD, Brown EW. Artificial Intelligence in Medical Practice: The Question to the Answer? Am J Med. Feb 2018;131(2):129-133.

18. Muller A, Mertens SM, Gostemeyer G, Krois J, Schwendicke F. Barriers and Enablers for Artificial Intelligence in Dental Diagnostics: A Qualitative Study. J Clin Med. Apr 10 2021;10(8). 
19. Chang HJ, Lee SJ, Yong TH, Shin NY, Jang BG, Kim JE, Huh KH, Lee SS, Heo MS, Choi SC, Kim TI, Yi WJ. Deep Learning Hybrid Method to Automatically Diagnose Periodontal Bone Loss and Stage Periodontitis. Sci Rep. May 5 2020;10(1):7531.

20. Sukegawa S, Yoshii K, Hara T, Yamashita K, Nakano K, Yamamoto N, Nagatsuka H, Furuki Y. Deep Neural Networks for Dental Implant System Classification. Biomolecules. Jul 1 2020;10(7).

21. Jaskari J, Sahlsten J, Järnstedt J, Mehtonen H, Karhu K, Sundqvist O, Hietanen A, Varjonen V, Mattila V, Kaski K. Deep Learning Method for Mandibular Canal Segmentation in Dental Cone Beam Computed Tomography Volumes. Scientific Reports. 2020;10(1).

22. Kwon JJ, Hwang J, Kim YD, Shin SH, Cho BH, Lee JY. Automatic three-dimensional analysis of bone volume and quality change after maxillary sinus augmentation. Clin Implant Dent Relat Res. Dec 2019;21(6):1148-1155.

23. Revilla-Leon M, Gomez-Polo M, Vyas S, Barmak BA, Galluci GO, Att W, Krishnamurthy VR. Artificial intelligence applications in implant dentistry: A systematic review. J Prosthet Dent. Jun 152021.

24. Nagrath P, Jain R, Madan A, Arora R, Kataria P, Hemanth J. SSDMNV2: A real time DNN-based face mask detection system using single shot multibox detector and MobileNetV2. Sustain Cities Soc. Mar 2021;66:102692.

25. Moon GC, Ju Yong; Lee, Kyoung Mu. V2V-PoseNet voxel-tovoxel prediction network for accurate 3d hand and human pose estimation from a single depth map. IEEE Conference on Computer Vision and Pattern Recognition 2018:5079-5088.

26. Chen Z, Li J, Sinjab K, Mendonca G, Yu H, Wang HL. Accuracy of flapless immediate implant placement in anterior maxilla using computer-assisted versus freehand surgery: A cadaver study. Clin Oral Implants Res. Dec 2018;29(12):1186-1194.

27. Vollmer S, Mateen BA, Bohner G, Kiraly FJ, Ghani R, Jonsson P, Cumbers S, Jonas A, McAllister KSL, Myles P, Granger D, Birse M, Branson R, Moons KGM, Collins GS, loannidis JPA, Holmes C, Hemingway H. Machine learning and artificial intelligence research for patient benefit: 20 critical questions on transparency, replicability, ethics, and effectiveness. BMJ. Mar 20 2020;368:16927.

28. Sondergaard K, Hosseini M, Jensen SS, Spin-Neto R, Gotfredsen K. Fully- versus conventionally guided implant placement by dental students. A randomized controlled trial. Clin Oral Implants Res. Jun 242021.

29. Vercruyssen M, Cox C, Coucke W, Naert I, Jacobs R, Quirynen M. A randomized clinical trial comparing guided implant surgery (bone- or mucosa-supported) with mental navigation or the use of a pilot-drill template. J Clin Periodontol. Jul 2014;41(7):717-723.

30. W. Liu DA, D. Erhan, C. Szegedy, S. Reed, C.-Y. Fu, et al. . SSD_ Single Shot MultiBox Detector. 14th Eur. Conf.Comput. Vis. Amsterdam, Netherlands: Springer-Verlag. 2016;p:21-37.

31. Wang YK, Syu HY, Chen YH, Chung CS, Tseng YS, Ho SY, Huang CW, Wu IC, Wang HC. Endoscopic Images by a Single-Shot Multibox Detector for the Identification of Early Cancerous Lesions in the Esophagus: A Pilot Study. Cancers (Basel). Jan 17 2021;13(2).

32. Yu Y, Liu Y, Chen J, Jiang D, Zhuang Z, Wu X. Detection Method for Bolted Connection Looseness at Small Angles of Timber Structures based on Deep Learning. Sensors (Basel). Apr 29 2021;21(9).

33. Orhan K, Bayrakdar IS, Ezhov M, Kravtsov A, Ozyurek T. Evaluation of artificial intelligence for detecting periapical pathosis on cone-beam computed tomography scans. Int Endod J. May 2020;53(5):680-689.

34. J. Y. Chang GMKML. V2V-PoseNet voxel-tovoxel prediction network for accurate $3 d$ hand and human pose estimation from a single depth map. IEEE/CVF Conference on Computer Vision and Pattern Recognition. 2018;p:5079-5088. 
35. Zhang X, Chen F, Yu T, An J, Huang Z, Liu J, Hu W, Wang L, Duan H, Si J. Real-time gastric polyp detection using convolutional neural networks. PLoS One. 2019;14(3):e0214133.

36. Datong C, Minghui L, Cheng J, Yue S, Dongbin X, Yueming L. Coronary Calcium Detection Based on Improved Deep Residual Network in Mimics. J Med Syst. Mar 25 2019;43(5):119.

37. Tahmaseb A, Wismeijer D, Coucke W, Derksen W. Computer Technology Applications in Surgical Implant Dentistry: A Systematic Review. The International Journal of Oral \& Maxillofacial Implants. 2014;29(Supplement):25-42.

\section{Figures}

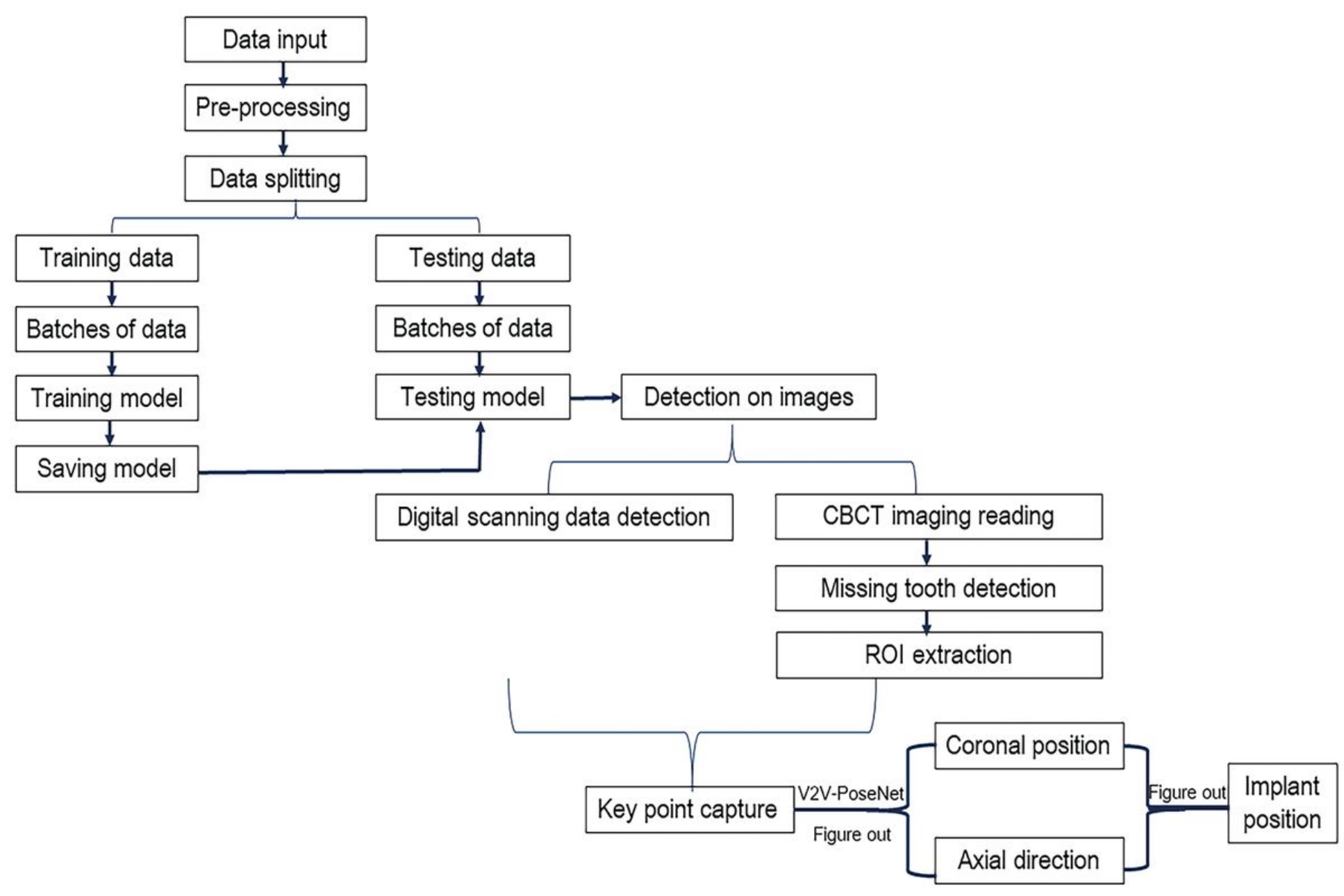

Figure 1

Al software workflow. 

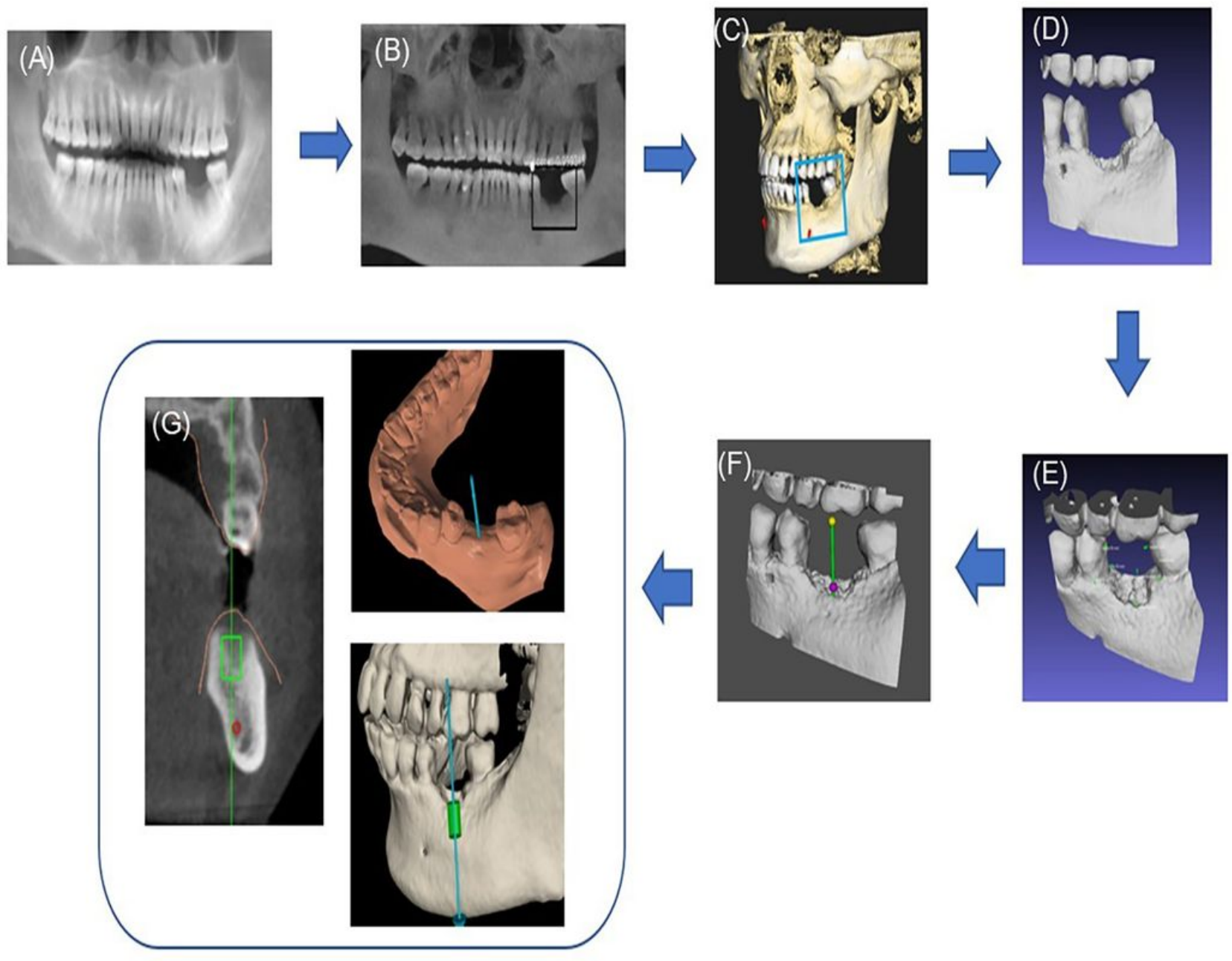

\section{Figure 2}

Image flow chart of automatically implant position design. (A) The panoramic image was created from the CBCT data. (B) The mandibular left first molar was detected based on SSD algorithm. (C) Construct a region of interest (ROI) from the CBCT data. (D) Detect the bone contour from the CBCT data based on V2V-PoseNet. (E) Capture and study the designed characteristic annotators through V2V-PoseNet. (F) Generate the implant axis based on the characteristic annotators. (F) Implant position automatically generated. 

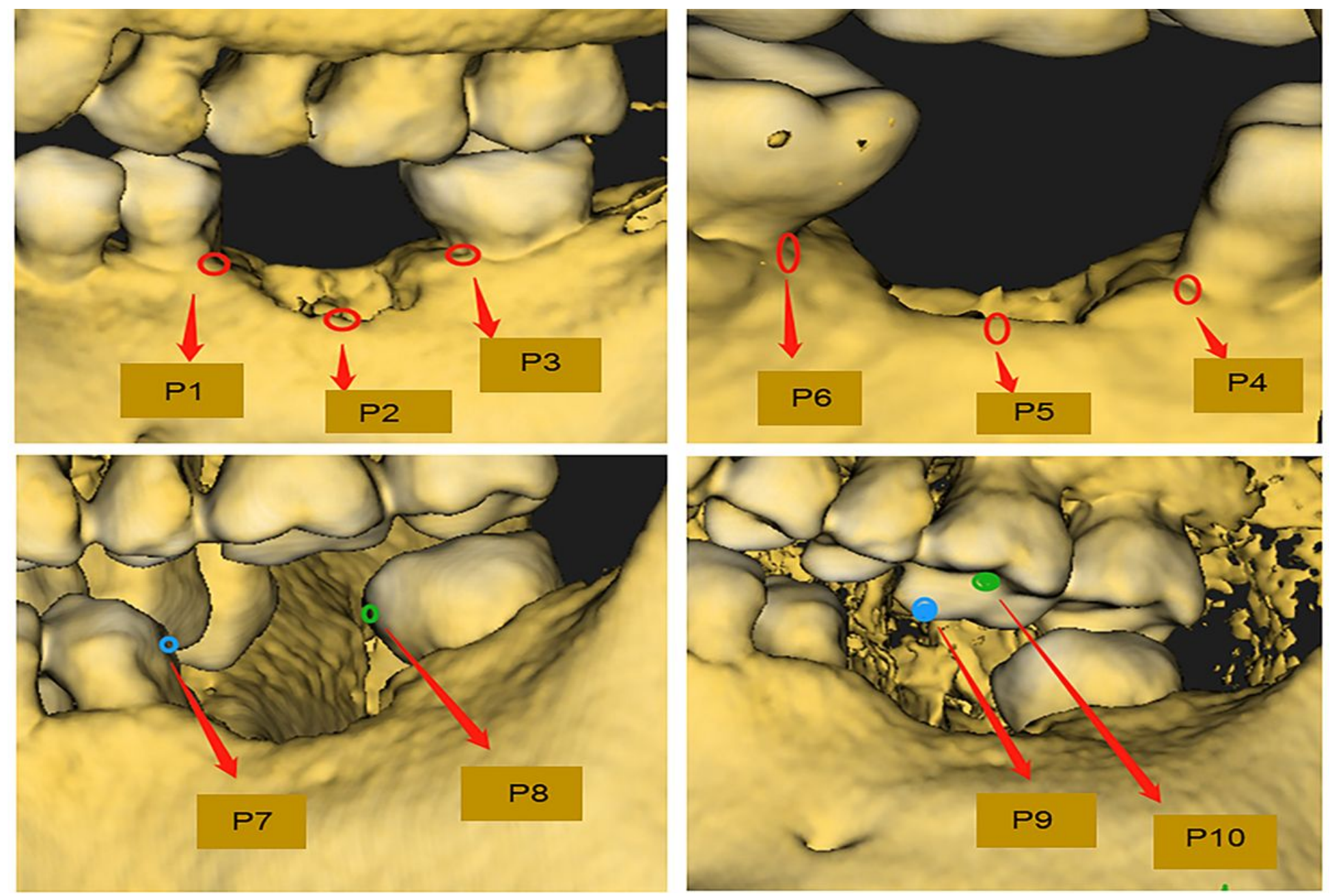

Figure 3

Diagram of the characteristic points. 


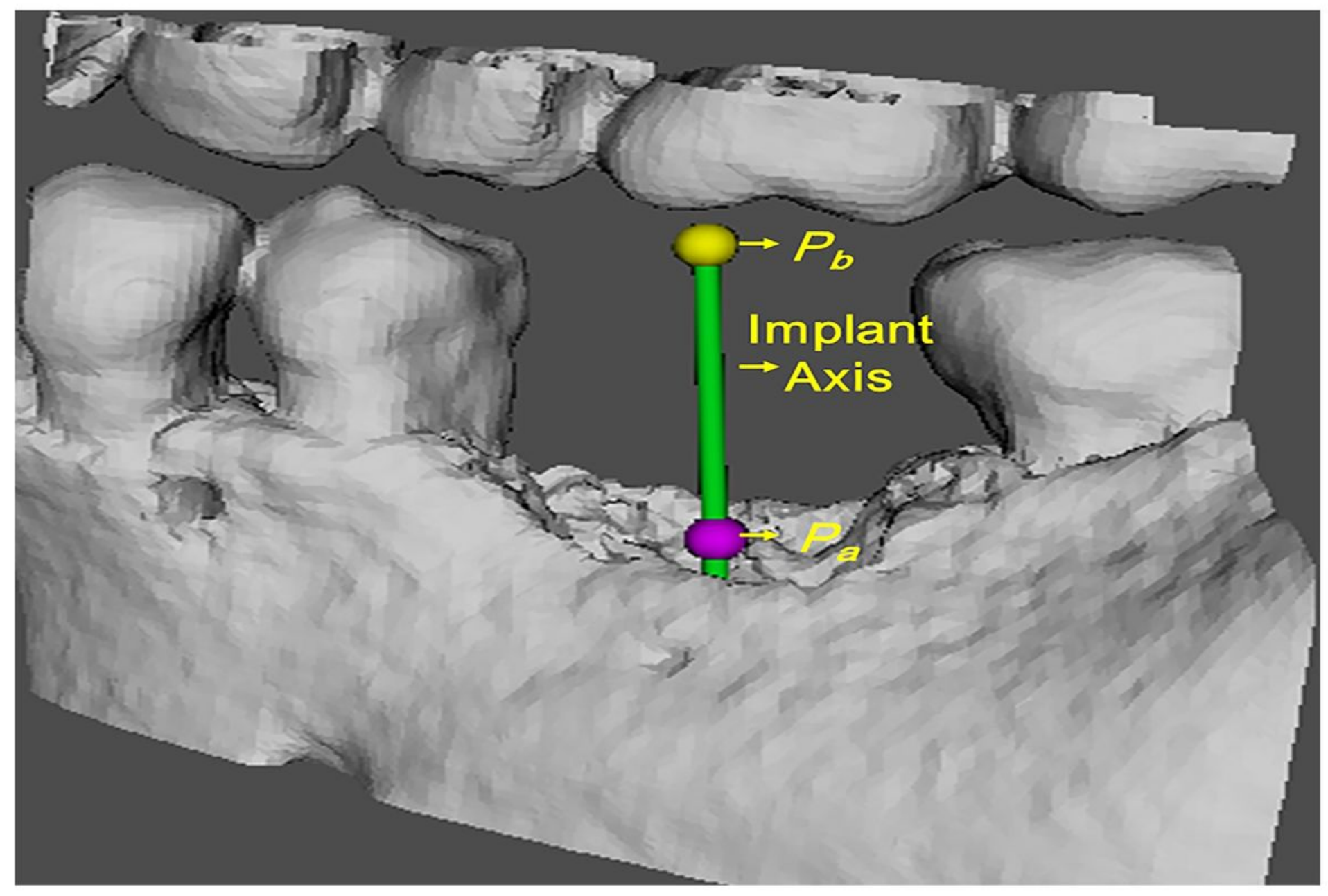

Figure 4

Diagram of three measurements ( $\mathrm{Pa}, \mathrm{Pb}$ and Implant axis). 


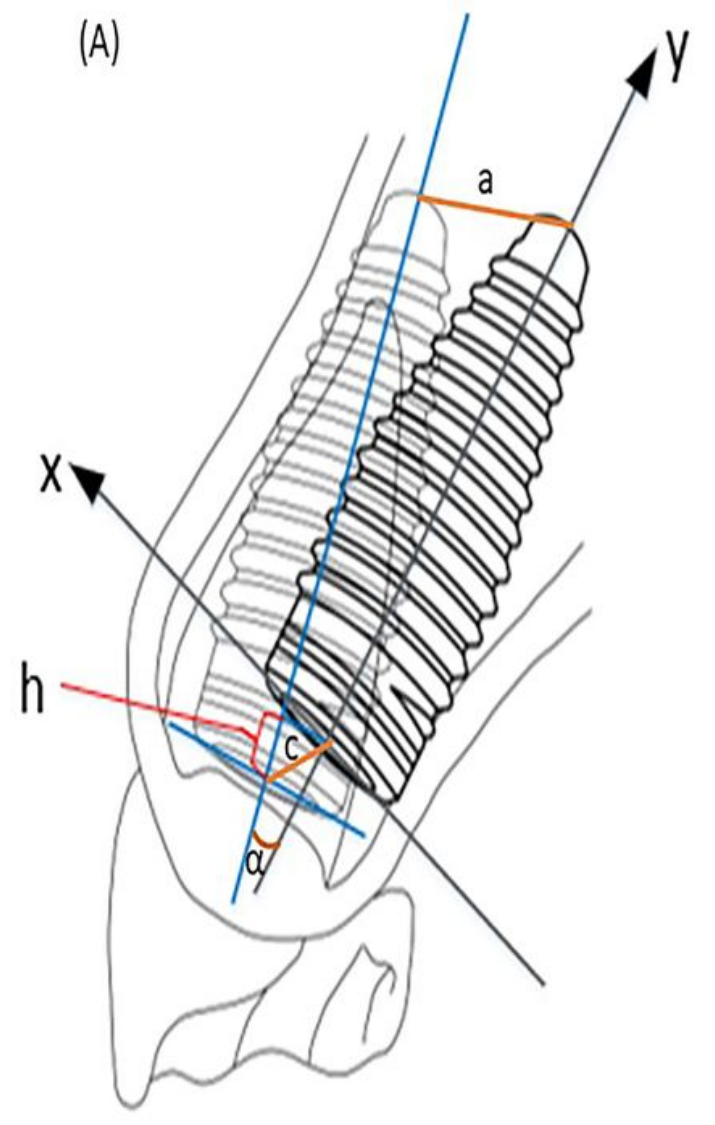

Depth, angular

\& global deviation

(B)

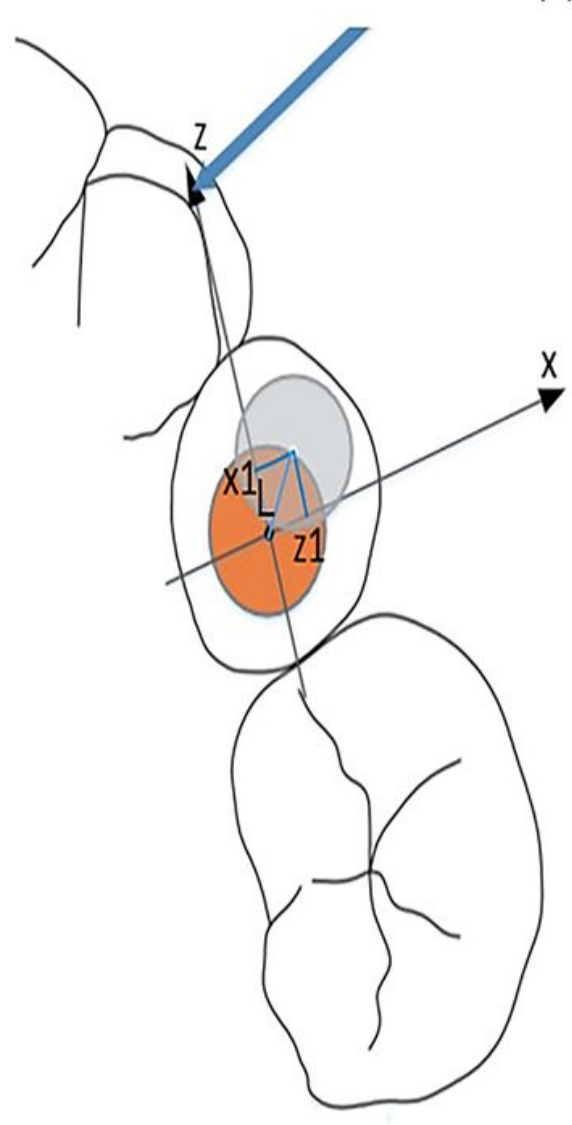

Lateral deviation (c)

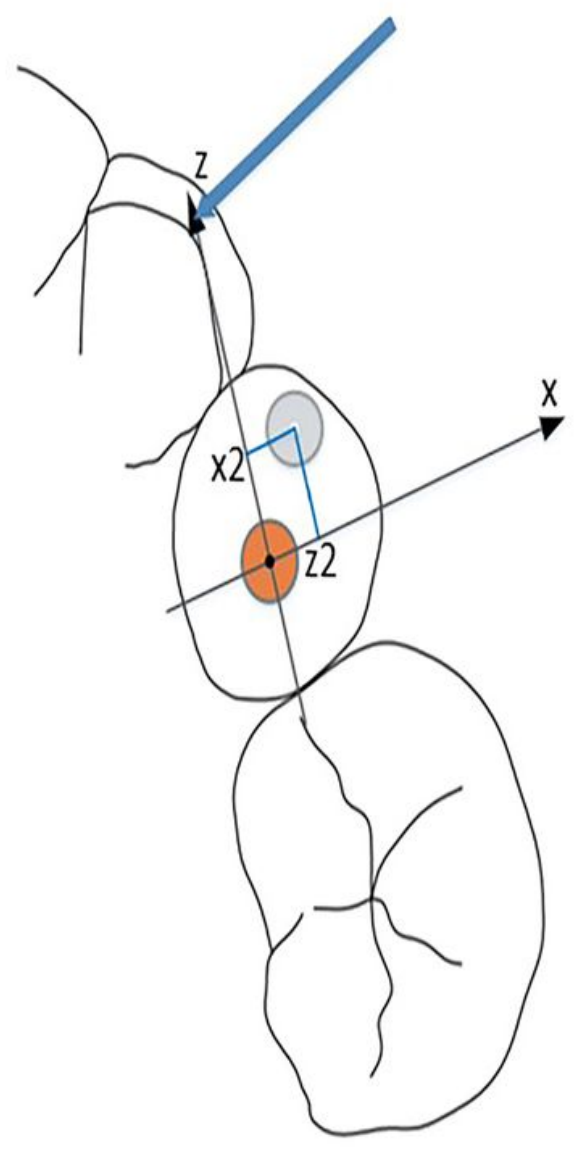

Apical deviation

\section{Figure 5}

Measurement of deviations between Al designed and ideal implant. (A) Depth, angular \& global deviation: h: depth deviation; a: angular deviation; global deviation (c: coronal deviation; a: apical deviation);(B) L: lateral deviation (x1: coronal bucco-lingual deviation; z1: coronal mesio-distal deviation); (C) Apical deviation (x2: apical bucco-lingual deviation; z2: apical mesio-distal deviation). 


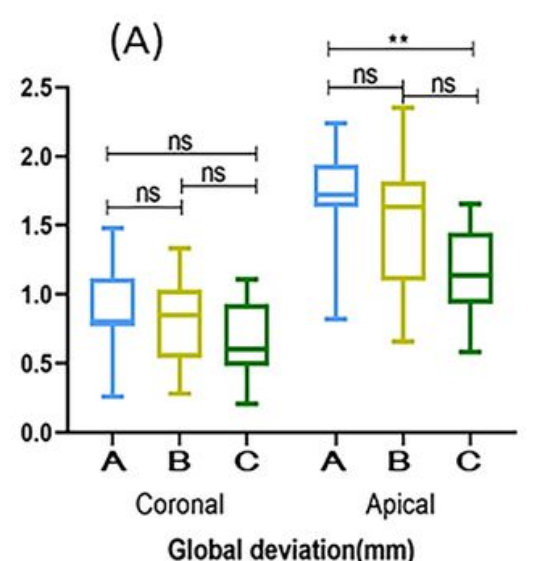

Global deviation(mm)

(D)

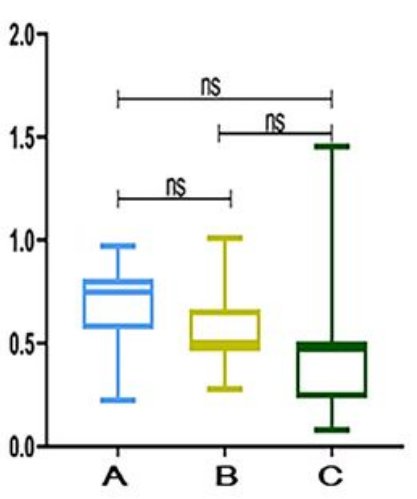

Lateral deviation(mm)
(B)

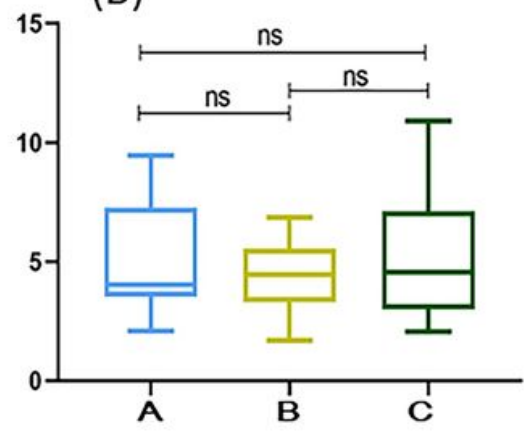

Angular deviation $\left({ }^{\circ}\right)$
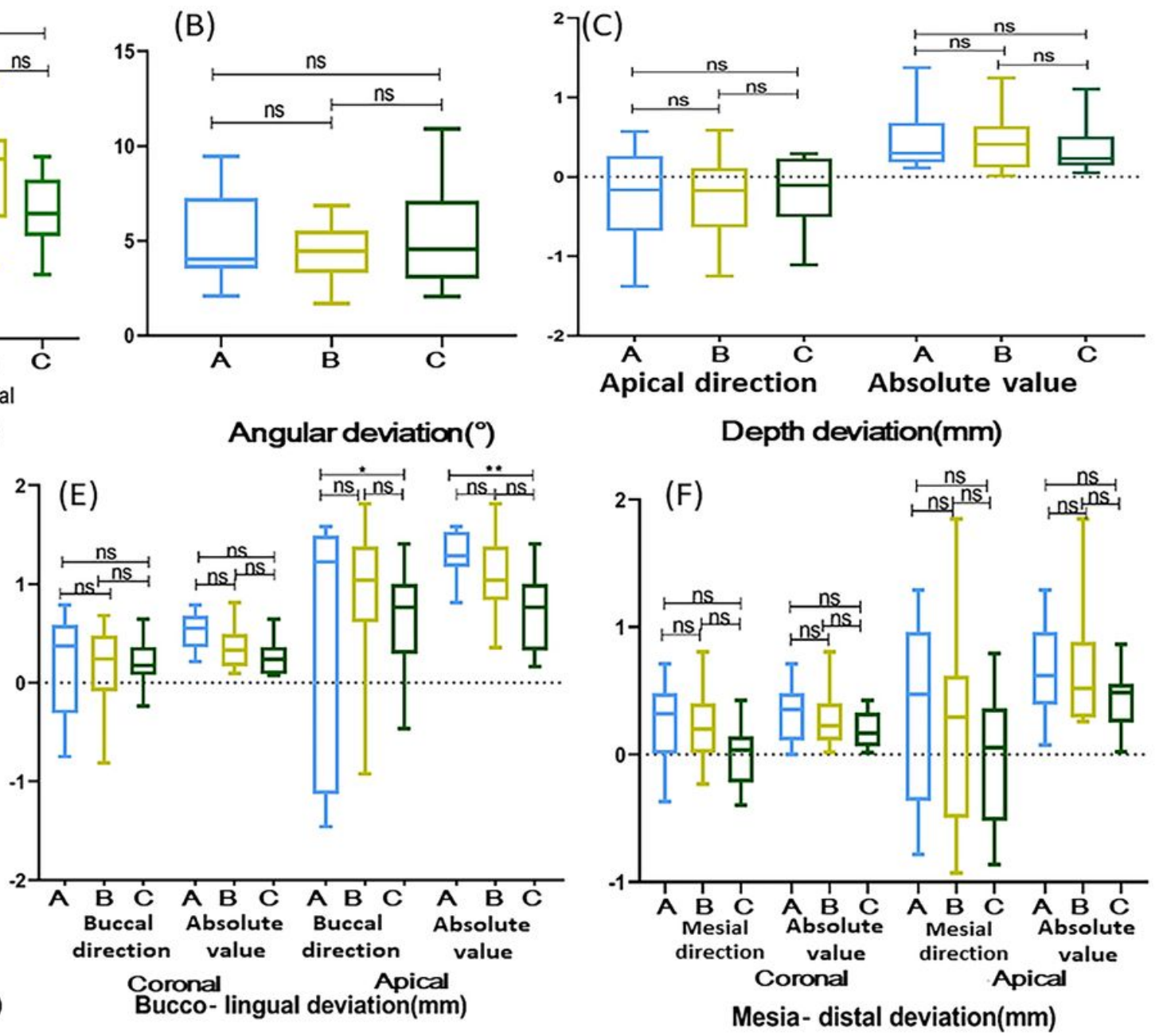

Figure 6

Deviation parameters in the three group. (a) Global deviations (coronal and apical); (b) Angular deviation; (c) Depth deviation (apical direction and absolute value); (d) Lateral deviations; (e) Bucco-lingual deviations (buccal direction and absolute value) at coronal and apical level; (f) Mesio-distal deviations(mesial direction and absolute value) at coronal and apical level. 